\title{
INVESTIGACIONES
}

\section{Los problemas del stock en campos profesionales difusos: Oferta educativa en Trabajo Social}

The problems of stock in fuzzy professional fields: Educational offer in Social Work Programs

\author{
Os problemas de estoque em campos profissionais difusos: \\ Oferta educativa em Serviço Social
}

\author{
Víctor Castillo Riquelme ${ }^{1}$, Carlos Rodríguez Garcés ${ }^{2}$ \\ 'Centro de Intervención e Investigación Social de la Escuela de Trabajo Social (CIISETS), \\ Cátedra Metodología de Investigación Social, Universidad del Bío-Bío \\ ${ }^{2}$ Departamento de Ciencias Sociales de la Universidad del Bío-Bío; Centro de Informática Educativa (CIDCIE)
}

\section{RESUMEN}

La desregulación de la oferta de programas formativos en Chile ha impactado en todas las áreas del conocimiento disciplinar y de la formación técnica. Estos efectos se han hecho particularmente visibles en la caso del Trabajo Social, disciplina que dada sus debilidades identitarias se presenta como más vulnerable a los juegos del mercado laboral y educativo. Este artículo, por medio de la revisión de las bases históricas del Consejo Nacional de Educación (CNED), analiza las principales problemáticas que evidencia la oferta educativa de la profesión, tales como la inminente tecnificación disciplinar, la saturación y multicompetencia laboral, así como las debilidades del resguardo del estatus profesional por la vía de la exclusividad universitaria que desde 2005 ostenta el título del Trabajo Social.

Palabras clave: trabajo social, oferta educativa, tecnificación disciplinar, stock profesional, exclusividad universitaria.

\section{ABSTRACT}

The deregulation of the offer of training programs in Chile has affected all areas of the disciplinary knowledge and technical training. These effects have been particularly visible in the case of Social Work, a discipline that given its weaknesses regarding identity is presented as more vulnerable to the variables of the job and educational market. This article -through a review of the historical foundations of the National Board for Education (Consejo Nacional de Educación-CNED) -analyzes the main problems evidenced by the educational offer of the profession, such as the imminent disciplinary modernization, saturation and labor multi-competition, as well as the weaknesses related to safety of the professional status by means of university exclusivity that, since 2005, holds the degree of Social Work.

Key words: social work, educational offer, disciplinary modernization, professional stock, university exclusivity.

\section{RESUMO}

A desregulamentação da oferta de programas formativos no Chile tem impactado todas as áreas do conhecimento disciplinar e de formação técnica. Estes efeitos têm sido particularmente visíveis no caso do Serviço Social, disciplina que, dada as suas debilidades identitárias, apresenta-se como a mais vulnerável no jogo dos mercados de trabalho e educativo. Por meio da revisão das bases históricas do Conselho Nacional de Educação (CNED), analisam-se as principais problemáticas que evidenciam a oferta educativa da profissão, tais como a iminente 
tecnificação disciplinar, a saturação e multicompetência laboral assim como as debilidades do resguardo do status profissional por meio de exclusividade universitária que, desde 2005, ostenta o título de Serviço Social.

Palavras-chave: serviço social, oferta educativa, tecnificação disciplinar, estoque profissional, exclusividade universitária.

\section{DEBILIDADES DE LA FORMACIÓN PROFESIONAL EN CHILE}

Hasta 1980 el sistema de educación superior chileno estaba compuesto exclusivamente por un reducido número de universidades con sedes en distintas ciudades del país. Si bien algunas de ellas habían surgido bajo el alero de organizaciones privadas, todas contaban con financiamiento público.

En este mismo año las políticas educativas amparadas en la configuración de un nuevo marco normativo dieron pie a un proceso de reforma que incluyó cambios significativos en la estructuración del sistema de educación superior. Estructuración que en años posteriores a la dictadura cívico-militar se fue consolidando, profundizando e incluso legitimando a través de un sucesivo número de cuerpos legales sin modificar esencialmente la concepción ideológica subyacente. Esta reforma permitió ampliar la participación en el mercado educativo y diversificar el sistema a través de la creación de nuevas Instituciones de Educación Superior (IES), Institutos Profesionales (IP), Centros de Formación Técnica (CFT) y Universidades privadas independientes. Esta actuación estuvo motivada, en parte, por la pretensión de descomprimir la creciente demanda por estudios universitarios, incorporar la formación para el trabajo a la educación formal y por sobre todo, la instalación de la lógica neoliberal de la política educativa que incentiva la participación del oferente privado en la provisión de servicios. Como consecuencia, a partir de los años 80, se acelera el crecimiento de la oferta de programas de estudio y el número de instituciones de nivel superior así como el volumen de la matrícula y cobertura.

El nuevo escenario de la educación superior reconoció formalmente tres tipos de certificaciones académicas: títulos técnicos de nivel superior, títulos profesionales y grados académicos (licenciado, magister y doctor). Las instituciones universitarias están facultadas para otorgar toda clase de títulos y grados. Los IP, por su parte, otorgan títulos técnicos de nivel superior y títulos profesionales, exceptuando un limitado grupo de 18 profesiones que, definidas por ley, su certificación requiere estar previamente en posesión del grado de licenciado y, en consecuencia, están reservados únicamente para las universidades ${ }^{1}$. Los CFT, en cambio, sólo se encuentran habilitados para entregar títulos técnicos de nivel superior.

Uno de los cambios más significativos observados en el proceso de reforma educativa ha sido la proliferación de instituciones de educación superior de variada índole, heterogéneamente distribuidas por el territorio nacional y de naturaleza especialmente privada, las cuales participan del mercado educacional otorgando licenciaturas en las distintas áreas del conocimiento disciplinar, títulos profesionales de formación general para el desempeño laboral, así como certificaciones técnicas de nivel superior de apoyo al estamento profesional. Con ello, las instituciones de educación superior buscan impactar sobre la demanda, aumentando, diversificando y flexibilizando la oferta educativa a través de la apertura de un sinnúmero de programas formativos.

\footnotetext{
En un comienzo la legislación resguardó la exclusividad universitaria de 12 carreras. Al 2013, la legislación chilena reconoce un total de 18 profesiones entre las que se incluye Trabajo Social, quien recupera el rango universitario el año 2005.
} 
Esta masificación de instituciones y programas permitió elevar significativamente las tasas de cobertura, alcanzando en la actualidad niveles históricos, de forma tal que uno de cada tres jóvenes entre los 18 y 24 años asiste o ha asistido a una IES, exhibiendo tasas de cobertura similares al promedio de los países de la OCDE (2009). Si bien el acceso a la educación superior se democratiza por cuanto siete de cada diez estudiantes son primera generación, esta mayor cobertura se hace en base a una fuerte segmentación socioeducativa, la reducción de los niveles de selectividad y calidad de los procesos formativos, así como al fuerte endeudamiento de los estudiantes o sus familias para financiar los altos costes que esta educación conlleva.

La educación superior, así como la generalidad del sistema educativo en Chile, está conscientemente estructurada por clases sociales (OCDE, 2009), con clusters cerrados, excluyentes y con una profunda homogeneidad interna en cuanto al origen social e incluso académico de sus estudiantes. Segmentación socioeducativa que no es más que el correlato de la segregación territorial y desigualdad social existente en Chile.

Se reducen los niveles de selectividad y se cuestionan fuertemente los sistemas de aseguramiento de la calidad en la labor formativa. En efecto, la diversificación de la oferta educativa implicó, como estrategia de segmentación de mercado, la necesaria flexibilización de los requisitos de ingreso, otrora regulados por un Sistema Único de Admisión. En base a este parámetro, el sistema de educación superior, en especial el universitario, se dualiza coexistiendo un reducido grupo de instituciones selectivas con un amplio número de universidades no selectivas y de masificada matrícula, con independencia relativa del área del conocimiento o disciplinar que aborden. Esto impacta en las condiciones de educabilidad o perfil escolar del estudiante que ingresa a la educación universitaria, dificultando la labor formativa en instituciones donde la atención a los problemas de aprendizaje, así como el remediar los profundos déficits de contenidos no conforman parte de su misión intrínseca.

No obstante lo anterior, el problema de la calidad no se limita ni explica únicamente por el perfil del estudiante universitario que ingresa a instituciones escasamente selectivas. El sistema educacional chileno presenta dentro de sus principales características fuertes problemas de calidad y pertinencia, donde las instituciones de educación superior, universidades incluidas, no son la excepción. Muy por el contrario, en la educación superior, en su afán por masificarse, proliferan instituciones y programas de cuestionable calidad, incapaces de administrar adecuadamente el currículum y proporcionar valor agregado a sus estudiantes como resultado de la acción formativa. Esta problemática cuestiona fuertemente a la generalidad del sistema de educación superior, articulándose una incipiente institucionalidad que busca supervisar, regular y certificar la acción formativa ${ }^{2}$.

La masificación y aumento de cobertura de la educación superior, junto con un déficit de calidad en la labor formativa, trae aparejado un problema de pertinencia y eventual sobrestock profesional. En efecto, el aumento de la dotación profesional y técnica que la masificación de la educación terciaria ha suscitado, así como la escasa planificación en la provisión de los servicios educativos, no pocas veces como efecto deliberado de la lógica liberal, inserta problemáticas latentes en la valoración profesional en el mundo del trabajo. La alta demanda por la obtención de capital humano en el mercado de educación superior ha propiciado la concentración de la matrícula en ciertas ramas y especialidades, ignorando

A modo de ejemplo: Agencia de Calidad, Superintendencia de Educación Superior, Programa INICIA, Comisión Nacional de Acreditación, Sistema de Aseguramiento de la Calidad. 
así las necesidades sociales y las tendencias de los mercados del trabajo que el país requiere para su desarrollo. Aunque esto es todavía un tema controversial, el explosivo aumento en el número de titulados que egresan de la educación superior generaría un sobrestock de profesionales que presionaría el mercado del trabajo con efectos en la disminución de los salarios, precarización del empleo, reducción de las tasas de empleabilidad, aumento de la subutilización del capital humano, así como la depreciación de la rentabilidad educativa (Rama, 2009; Meller y Rappoport, 2003; Camarena y Velarde, 2009). Este desajuste entre la oferta de vacantes en el nivel superior y las reales posibilidades de absorción del contingente de titulados por el mercado laboral termina afectando las condiciones de empleo, conminando a profesionales a subemplearse en actividades que poco tienen que ver con la formación profesional recibida. Residualmente, se instala una mayor presión por la sobrecapacitación a través de estudios adicionales que certifiquen competencias para una mejor inserción laboral. El significativo aumento en la matrícula de postgrado sería también expresión de este fenómeno. En consecuencia, asistiríamos a un escenario de sobrestock educativo que detona problemas de vinculación consistente y de responsabilidad social por parte de las instituciones formadoras, expresión de una débil articulación con el mundo del trabajo. Es decir, la desatención del necesario vínculo de pertinencia entre el proceso formativo y las prácticas laborales, para centrar sus esfuerzos en la generación artificiosa de una demanda por medio del marketing y el aumento de sus utilidades por parte de las IES.

En sintonía con lo anterior, se evidenciaría una suerte de depreciación del valor de la educación universitaria en determinadas profesiones respecto de carreras similares o afines ofrecidas por IP y CFT. Aunque con matices y sesgos según el campo disciplinar que se trate, los diferenciales de renta entre formación universitaria e instituto profesional se atenúan haciendo comparativamente poco rentables las mayores inversiones de tiempo y dinero que la educación universitaria conlleva, observándose además que los niveles de renta de determinadas profesiones universitarias sean incluso inferiores al de carreras técnicas de nivel superior.

Esta pérdida de estatus que otrora gozaba la educación universitaria impacta en todas las áreas del saber, aunque con rezagos en aquellos contextos disciplinares que por tradición o fuerza gremial parecían más fortalecidos, como sería el caso de las carreras del área de la salud. Evento que se suscita con independencia relativa del resguardo institucional brindado a determinadas profesiones asignándoles el rango universitario. En efecto, en Chile existe un total de 18 profesiones exclusivamente universitarias, rango otorgado como forma de resguardar la calidad y rigor disciplinario que precisaban estos oficios. Este mecanismo ha sido fuertemente resistido por algunos sectores, en especial instituciones privadas de carácter no universitario, por cuanto operaría con cierta independencia del estatus social o prestigio académico que en esencia determinadas profesiones requerirían, constituyéndose en una lista arbitraria más influenciada por la presión de diferentes asociaciones gremiales que por la naturaleza científica intrínseca de los títulos, vulnerando la libertad de enseñanza al discriminar a los institutos profesionales (OCDE, 2009).

No obstante lo anterior, en la práctica la utilidad de este mecanismo de exclusividad ha quedado amortiguada por medio de la implementación de un conjunto de estrategias de cooperación vertical entre instituciones de un mismo controlador, así como por la generación de programas técnicos y profesionales de denominaciones diversas pero homologables, logrando de esta manera que las instituciones de carácter no universitario se acomoden al sistema (Brunner, 2006) 
En el contexto de la educación superior, existen holdings educativos que integran un conjunto diversificado de instituciones en manos de un mismo controlador que concentra una amplia matrícula, situación que les posibilita como estrategia de negocio segmentar su oferta educativa, articulando verticalmente los distintos niveles de educación superior como un continuиm por el cual transita un contingente no despreciable de su demanda cautiva. Entre las prácticas recurrentes para la continuidad de estudios de nivel superior está la convalidación de asignaturas, la reducción de arancel, la disminución del número de años y la creación de programas especiales de licenciatura a los egresados de institutos profesionales.

Así también, sea como estrategia para diferenciarse en el mercado de la demanda educativa o como forma de vulnerar restricciones que regulan determinados títulos profesionales, las instituciones de carácter no universitario han propiciado la creación de un conjunto no despreciable de denominaciones afines en un esfuerzo por emular a aquellas profesiones a las que su oferta les es proscrita. A modo de ejemplo, en el ámbito del Trabajo Social en la formación Técnica de Nivel Superior, encontramos 12 denominaciones.

Las problemáticas, vicios y debilidades del sistema de educación superior en Chile no le son ajenas al Trabajo Social, sea entendida como sub-área de conocimiento disciplinar o en su carácter de formación profesional de rango universitario. La existencia de un mercado educacional fuertemente desregulado, la presión de los grupos sociales medios y bajos para acceder a la educación superior, así como la existencia de un diversificado y extendido conjunto de instituciones de educación superior, han impactado fuertemente en la oferta de programas formativos asociados al Trabajo Social. Es por tanto propósito de este artículo dar cuenta de las principales tendencias asociadas en la oferta programática del área del Trabajo Social, destacando la inminente tecnificación de la profesión y el presumible superávit de profesionales en competencia por el mismo campo laboral.

\section{MATERIAL Y MÉTODO}

Los resultados de esta investigación fueron obtenidos a partir de la bases de datos INDICES de Pregrado, provista por el Consejo Nacional de Educación (CNED) ${ }^{3}$ para los años 1997-2013, las cuales proporcionan información actualizada respecto de la matrícula y programas tanto técnicos como profesionales de las Instituciones de Educación Superior en Chile. Respecto de la base de datos es menester advertir tres observaciones de eventual relevancia, a saber:

- En primer lugar, si bien se trata de bases de datos oficiales y utilizada por los organismos competentes para la generación de informes, proyección de políticas u otras acciones afines, la información es autoreportada voluntariamente por las IES; ello puede explicar la ausencia de registros en algunos campos, eventualmente considerados sensibles para la imagen institucional.

- $\quad$ En segundo lugar, sólo a partir del año 2005 el CNED incorpora en sus bases la información que proveen los CFT, por lo que no es posible realizar comparaciones entre los tres niveles del sistema anteriores a dicha fecha.

- Finalmente, las bases no consignan información respecto de los programas especiales desarrollados por la Universidad de los Lagos para el período 2006-2008, así como tampoco información de los programas de la Universidad del Mar para el año 2013, por encontrarse en proceso de cierre. 
El análisis de los datos realizados por medio del software estadístico SPSS, en su versión 15.0, da cuenta de la existencia de un total de 2.898 programas vinculados al subsector del Trabajo Social para el cohorte 1997-2013; programas que son ofertados en sus diversos niveles a lo largo del territorio nacional por instituciones de educación superior tanto públicas como privadas. A partir del 2005, a los ya usuales Institutos Profesionales y Universidades, se incorpora en el registro la oferta educativa proporcionada por los Centros de Formación Técnica, cuya información anterior a dicha fecha el CNED no consignaba.

Cuadro $N^{\circ} 1$. Caracterización de la muestra

\begin{tabular}{|c|c|c|}
\hline & Programas (n) & Matrícula total \\
\hline 1997 & 31 & 5.296 \\
\hline 1998 & 34 & 6.087 \\
\hline 1999 & 45 & 6.518 \\
\hline 2000 & 53 & 6.935 \\
\hline 2001 & 63 & 10.647 \\
\hline 2002 & 70 & 11.638 \\
\hline 2003 & 80 & 12.942 \\
\hline 2004 & 127 & 13.679 \\
\hline 2005 & 171 & 15.993 \\
\hline 2006 & 223 & 16.240 \\
\hline 2007 & 213 & 16.547 \\
\hline 2008 & 225 & 17.739 \\
\hline 2009 & 251 & 20.705 \\
\hline 2010 & 267 & 23.999 \\
\hline 2011 & 325 & 27.466 \\
\hline 2012 & 343 & 29.656 \\
\hline 2013 & 377 & 31.460 \\
\hline Total & 2.898 & 273.547 \\
\hline
\end{tabular}

Fuente: CNED - INDICES, Elaboración propia

Si bien en el análisis se desagrega expresamente al Trabajo Social en su componente profesional y/o carácter universitario, también se hace mención a la situación de la formación Técnica de Nivel Superior de denominaciones afines, siendo además agrupada bajo el rótulo "sub-área del Trabajo Social", cuyo conjunto de programas integran la unidad de observación o análisis.

La pertinencia de considerar como unidad de análisis a todos los programas agrupados en la sub-área Trabajo Social radica en la escasa discriminación que hace el mercado laboral 
a la hora de ofertar puestos de trabajo y en el solapamiento teórico de los límites de las carreras sociales. En este sentido Iturrieta (2012) expone la tendencia del mercado laboral chileno de sustituir las ofertas dirigidas a profesionales específicos por aquellas bajo el rótulo de profesional de las ciencias sociales evidenciando por tanto, un campo profesional con límites difusos que confunden los ámbitos disciplinares, así como la distinción de la experticia técnica o profesional requerida para un puesto de trabajo.

\section{ANÁLISIS DE DATOS}

\subsection{LA MASIFICACIÓN DE LA OFERTA EDUCATIVA DE NIVEL SUPERIOR}

La reforma al sistema de educación superior implementada a partir de los años ochenta sentó las bases político-jurídicas para la apertura del mercado al oferente privado, que entre sus efectos más visibles implicaría la fuerte irrupción de programas e instituciones de educación superior.

Hasta el año 1980, existían en Chile un total de ocho universidades, las estatales y públicas concentraban el $65 \%$ de la matrícula y el sistema registraba una cobertura para la población entre 18 y 24 años del orden del 7,2\% (OCDE, 2009). Como resultado de la acción de la reforma educativa, para el año 2013 existen un total de 147 IES, entre CFT, IP y Universidades. Si bien su explosivo crecimiento se ha estabilizado producto de las dinámicas de ajuste por fusión y cierres, éstas han operado desde la lógica de la autorregulación y del mercado más que desde el accionar estatal de la política educativa.

Para el año 2012, el sistema de educación superior registra una matrícula de alrededor de 1.127.200 estudiantes, alcanzando una cobertura bruta para el rango etario de 18 a 24 años del $54,6 \%$.

El sistema de educación superior se masifica con independencia relativa del sector social de procedencia, pero de una calidad fuertemente segmentada en base al origen social del alumnado. En el lapso de una generación, los porcentajes de matrícula de estudiantes de los dos quintiles de ingreso más bajo, han aumentado en un factor de cinco, pero tienen los peores puntajes de ingreso, los mayores niveles de deserción así como mayores probabilidades de tener que pagar el costo de sus estudios (OCDE, 2009).

Esta expansión del sistema de educación superior, a lo largo y ancho del territorio nacional, en oleadas sucesivas, impacta sobre todas las áreas del conocimiento y de la formación profesional, aunque no necesariamente de forma homogénea en su distribución espacial y temporal. Así por ejemplo, en un primer momento, las nuevas universidades privadas, en la medida que tenían la supervisión del Consejo Superior de Educación, mantenían un stock limitado de programas circunscritos a un mismo espacio territorial donde operaba la casa matriz o central; para en un segundo momento, una vez obtenida la autonomía, expandir y diversificar su oferta educativa. La racionalidad operante buscaba aprovechar los vacíos de cobertura en las cabeceras de provincia para instalar sus sedes y abrir un conjunto de programas en razón del prestigio social de la profesión ofertada y los costos que su implementación implicaba. Ello explicaría el por qué las carreras asociadas al ámbito de la salud, con costos de implementación significativamente mayores, sean las últimas en masificar su oferta, fenómeno que acontece una vez instalada la industria educativa (figura $\mathrm{N}^{\circ} 1$ ). 
Figura $N^{\circ} 1$. Evolución programas por área de conocimiento

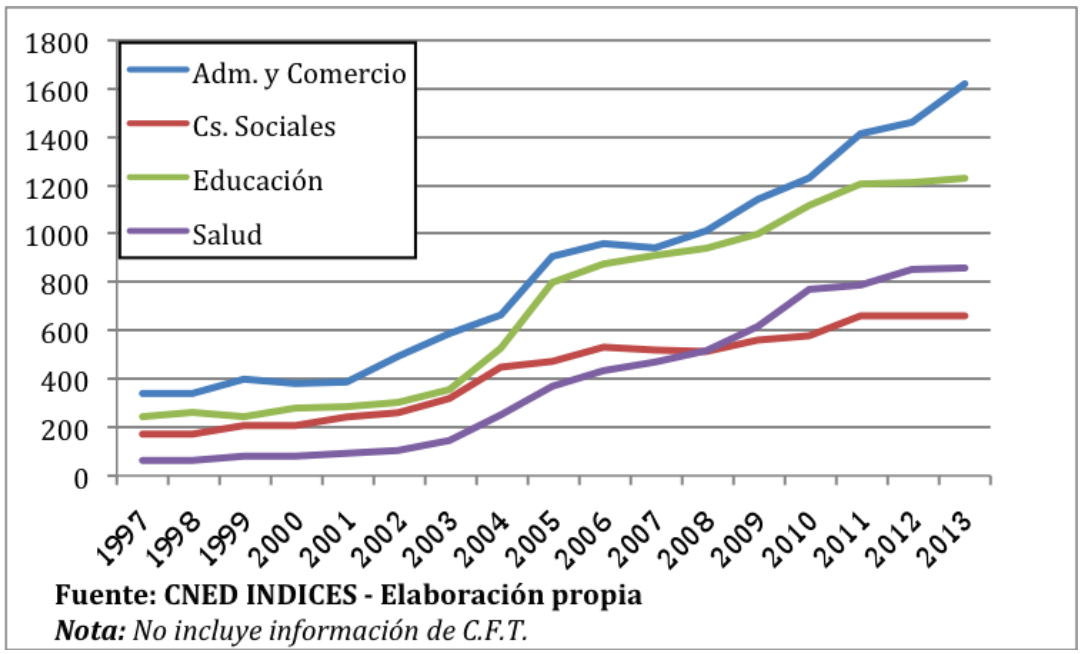

En efecto, la oferta educativa de programas de pregrado asociados al área de la salud observa en sus primeros años una tasa de crecimiento prácticamente nula, acelerando su ritmo a partir del 2003, y especialmente en el período 2008-2010, constituyéndose de paso en una de las áreas formativas donde la reforma educativa, en especial la participación del oferente privado, tardó más tiempo en manifestar su impacto. Costos de implementación, ausencia de campos clínicos, así como proteccionismos gremiales serían elementos que contribuyen a explicar este comportamiento.

La misma gráfica da cuenta del sostenido y fuerte crecimiento del área de Administración y Comercio, la cual año a año aumenta la oferta de programas a un ritmo superior al de otras áreas siendo seguida un poco más atrás por Educación. La gran demanda por estudios superiores, así como los bajos costes de entrega de formación de esta naturaleza, se traducen en una fuerte expansión de la oferta educativa, las tasas de matrícula y el número de egresados, dando lugar a una proliferación de programas e instituciones que ofrecen estos servicios educativos a un heterogéneo contingente de alumnos de capacidades y habilidades muy variadas, en correspondencia con los niveles de exigencia y calidad de los mismos procesos formativos. Mención especial merece aquí Educación, cuya matrícula se ha más que duplicado desde 2002 a la fecha. Crecimiento que no se condice con las necesidades que tiene el país de nuevos docentes atendiendo a las prácticamente universales tasas de cobertura de la educación básica y media, así como a la sostenida caída de la natalidad.

\subsection{STOCK Y MULTICOMPETENCIA PROFESIONAL EN EL TRABAJO SOCIAL}

El área de las Ciencias Sociales, sector disciplinar al cual pertenece el Trabajo Social, por involucrar exclusivamente a un set limitado de saberes asociados a las humanidades tales como Psicología, Trabajo Social, Antropología y Sociología, entre otros, evidencia comparativamente un nivel de crecimiento más restringido, expresión del heterogéneo comportamiento que tienen los elementos que la componen. 
Figura $N^{\circ}$ 2. Evolución Programas, Cs. Sociales

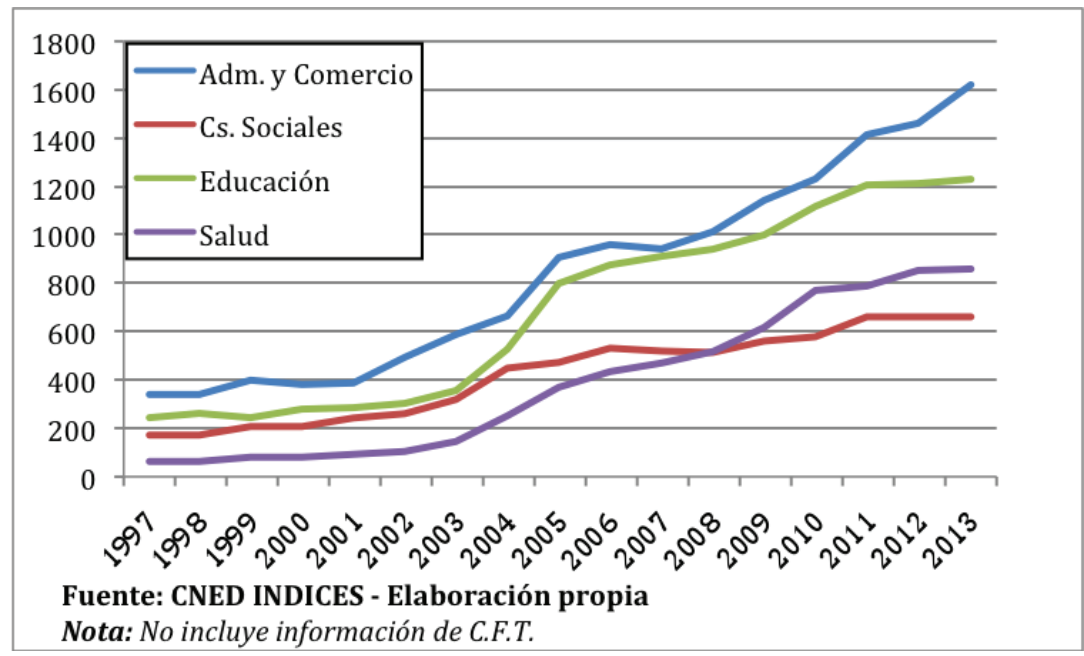

La figura $\mathrm{N}^{\circ} 2$ muestra el incremento de la oferta programática de las sub-áreas afines al Trabajo Social al interior de las Ciencias Sociales, en el entendido que son ramas que comparten un mismo campo disciplinar de carácter general y que, en el ámbito del desempeño profesional, interactúan cotidianamente.

Históricamente, el Trabajo Social al ser su objeto de acción la intervención social, ha registrado difusos límites profesionales. Esta nebulosa en torno a dónde empieza y termina lo que se puede definir como acción propia y exclusiva del quehacer profesional del trabajador social ha posibilitado la irrupción de otros perfiles profesionales en su mayor parte vinculados al área del conocimiento de las ciencias sociales -tales como Psicología, Antropología y Sociología-, quienes eventualmente compiten por un mismo mercado laboral. Incluso, aunque de presencia menos numerosa, esta permeabilidad también ha abierto la participación de otras disciplinas no necesariamente afines en lo que a perfil formativo se refiere. Se suma a ello un conjunto significativo de propuestas formativas que operan dentro del ámbito de la formación técnico profesional.

Dada la escasa claridad de los límites del quehacer profesional, cualquier análisis de la oferta formativa en Trabajo Social, así como de sus niveles de empleabilidad, precisan, en el contexto comparativo, abordar y referirse necesariamente al menos a la situación de las carreras afines que comparten una misma área formativa y evidencian un alto nivel de probabilidad de constituirse en competencia de un mismo campo laboral. En efecto, se ha vuelto un lugar común observar la cohabitación multiprofesional en una misma dependencia laboral bajo el rótulo genérico de profesional de las Ciencias Sociales. Situación especialmente notoria en el trabajo interdisciplinario de las duplas psico-sociales, donde trabajadores sociales y psicólogos conjugan sus saberes y experticia por un objetivo de intervención común.

La figura $\mathrm{N}^{\circ} 3$ muestra, en líneas de tiempo y cifras absolutas, la oferta programática de las subáreas de las Cs. Sociales, observándose un disímil comportamiento evolutivo. La oferta de programas formativos en Antropología y Sociología, en términos comparativos, es eminentemente más reducida y restringida, manifestando lentitud en sus procesos expansivos 
en la participación por una cuota de mercado en la formación en el área de las Cs. Sociales, segmento fuertemente dominado por Psicología y Trabajo Social. En efecto, la formación en Trabajo Social muestra un alto y sostenido crecimiento, especialmente visible a partir de 2003. Oferta fuertemente alterada por la irrupción de programas no universitarios de formación técnica o profesional, situación que no es extensible a las otras sub-áreas de las Cs. Sociales. No obstante la hegemónica dominancia de Trabajo Social, con tasas de crecimiento del orden del 16\% anual para el periodo 1997-2013, las otras sub-áreas igualmente se expanden, pero a tasas más lentas: Psicología, 6,4\%; Sociología, 5,9\%; Antropología, 4,9\%.

El aumento significativo de la oferta programática tiene, como es lógico, un efecto inmediato en la expansión de la matrícula, o de números de vacantes, ofrecida por el sistema de educación superior en las ramas de las Ciencias Sociales.

Figura $N^{\circ}$ 3. Evolución Matrícula, Cs. Sociales

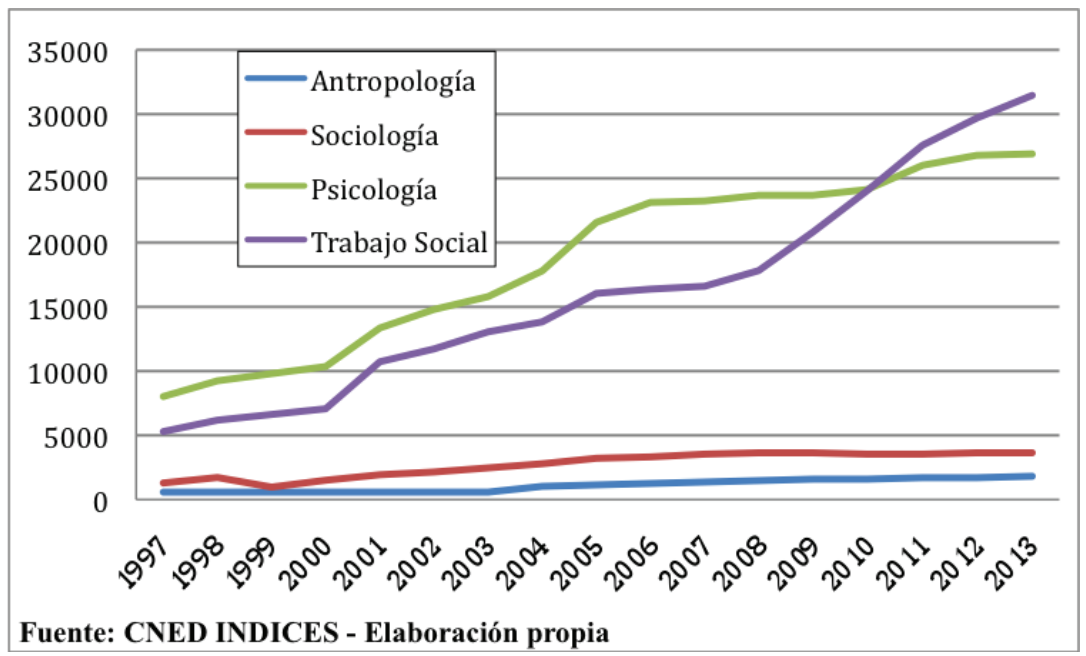

Se observa un aumento casi exponencial de la matrícula en las sub-áreas de Psicología y Trabajo Social. En los últimos dieciséis años, la matrícula en Psicología ha aumentado en un 235\%, pasando de 8.024 alumnos a tener en la actualidad 26.905. Si bien registra tasas de crecimiento relativo similares a las de Trabajo Social, su tendencia, a diferencia de este último, es a ralentizarse a partir del 2006. En efecto, Trabajo Social es una de las sub-áreas que más crece en términos comparativos. La matrícula en este sector en 1997 era de 5.296, cifra inferior a la de Psicología para igual período, para alcanzar en la actualidad a los 31.460. Es decir, Trabajo Social aumenta su matrícula en un factor de 5, creciendo a tasas del orden del $12 \%$ anual. Y si bien el perfil evolutivo de la matrícula siempre ha sido ascendente, éste se acelera significativamente a partir de 2007; crecimiento de la matrícula más asociado a la apertura y proliferación de sedes en el territorio nacional que a la creación de nuevas instituciones que participen de este mercado educacional.

El aumento exponencial que evidencian estas carreras de las Ciencias Sociales, en especial el de Psicología, se explica tanto por el prestigio social que ostenta, el cual influye 
sobre la demanda, como por el hecho de evidenciar menores costes de implementación. La expansión de esta oferta, sea en número de programas o de matrícula total, implicaría que cada año se incorpore un nuevo y numeroso contingente profesional a competir por un mismo campo laboral. Se estima que esta mayor disponibilidad de recursos humanos calificados generaría un sobrestock de capital humano avanzado que afectaría el valor relativo de la formación profesional recibida, con un impacto decisivo en los retornos de la inversión realizada dada la baja de los salarios. Deterioro en los retornos de los salarios, que sumado a la alta inversión realizada, generalmente en base al endeudamiento, perfilan un panorama sombrío y escasamente alentador. Si bien, esto es todavía un tema controversial por cuanto los datos de reconocidas investigaciones informan que se evidencia un sostenido aumento en los retornos de la educación superior en los salarios en detrimento de la valoración de la educación secundaria (Bravo \& Sanhueza, 2008; Beyer, 2000; Meller, 2010), estas mismas investigaciones no abordan las especificidades que se pudieran dar al interior de las diversas áreas formativas y campos profesionales determinados. Es decir, las tasas de retorno o rentabilidad educativa a través de los ingresos del trabajo tendrían comportamientos disímiles, tanto debido a la naturaleza de la formación recibida y la institución educativa, como a la valoración social y económica que hace el mercado del trabajo. Situación que sería especialmente preocupante en el caso de Trabajo Social, por cuanto sus espacios laborales no sólo tienden a ser compartidos con otras profesiones afines, sino que además debe enfrentar el numeroso contingente de nuevos egresados, así como las presiones de un sector técnico de nivel superior que se abre camino.

\subsection{TECNIFICACIÓN DISCIPLINAR Y EL AJUSTE DE LA OFERTA EDUCATIVA EN TRABAJO SOCIAL}

La atenuación de las fronteras profesionales no sólo se expresa en espacios laborales cada vez más traslapados con otras profesiones que se circunscriben dentro del perfil genérico de profesional de las Ciencias Sociales, sino que además este mismo campo de desempeño es compartido por un conjunto de otros oficios técnicos de nivel superior, siendo precisamente en el ámbito del Trabajo Social profesional donde esta situación de cohabitación se evidencia con más fuerza.

Es así como actualmente la sub-área del Trabajo Social destaca con un total de 377 programas educativos impartidos por 63 IES, que en términos desagregados corresponden a 39 Universidades, 14 Institutos Profesionales y 10 Centros de Formación Técnica. De la oferta implementada, 7 de cada 10 programas son de carácter no universitario $(69,5 \%)$. Mientras las universidades tradicionales del CRUCH se concentran en un espacio territorial históricamente delimitado, las instituciones educativas de carácter privado, en especial IP y Universidades, se extienden a lo largo del país. Hay instituciones de marcada presencia supra-regional, dando cuenta de un mercado que tiende fuertemente a la concentración horizontal y vertical. Es así como el 65\% de los programas de Trabajo Social son ofertados por tan sólo 10 de las 63 instituciones de educación superior que participan en esta área.

Sea producto de fusiones o expansión de sedes, hay un conjunto de instituciones que tienden a consolidarse fuertemente en el contexto nacional, concentran gran parte de la matrícula, y son actores dominantes en el mercado. Al 2013, existe un total de 31.460 alumnos matriculados en algún programa de Trabajo Social, perteneciendo un 42,5\% a universidades. La matrícula está fuertemente dominada por el sector privado, la de carácter público registra una marginal presencia del 13,1\% del alumnado. A esta desigual 
distribución del mercado educacional se suma el desequilibrado crecimiento que se evidencia en el espacio territorial, la región Metropolitana y del Bío-Bío concentrando el $32,7 \%$ y $18,0 \%$, respectivamente.

Cabe señalar que esta oferta programática, a diferencia de otras disciplinas como Psicología o Antropología, no se traduce únicamente en programas profesionales y/o de carácter universitario. Muy por el contrario, y tal como se ha hecho notar anteriormente, en el quehacer del Trabajo Social coexisten un conjunto de oficios y profesiones afines, y de rango técnico de nivel superior.

Amparado en esta situación es que, para fines de gestión de información, el CNED descompone las áreas del conocimiento en diversas dimensiones o sub-áreas. Dentro del área del conocimiento de las Ciencias Sociales, a nivel desagregado se encuentra la sub-área del Trabajo Social, segmento que agrupa al conjunto de programas afines a la disciplina o al campo profesional de diversa denominación y carácter. Es así como, además de la tradicional designación de Servicio Social o Trabajo Social, sea en su formato profesional o universitario, encontramos un conjunto no despreciable de programas de carácter técnico de diversificada denominación, tales como Ingeniería de Ejecución en Planificación y Desarrollo Social, Técnico Nivel Superior en Desarrollo comunitario, Técnico Nivel Superior en Administración de Programas Sociales, entre otros.

La sub-área del Trabajo Social experimenta un significativo incremento en términos de programas y matrícula ofrecida, constatándose un crecimiento del orden del $1.116 \%$ y $494 \%$, respectivamente, dentro del periodo analizado, con tasas de crecimiento a nivel de matrícula del orden del $12 \%$ anual, situándola dentro de las de mayor expansión en el contexto nacional, especialmente en el último decenio.

Cuadro $N^{\circ}$ 2. Evolución Matrícula en Trabajo Social según características institucionales.

\begin{tabular}{|l|c|c|c|c|c|c|c|c|c|c|c|}
\hline & \multicolumn{2}{|c}{2005} & \multicolumn{2}{c|}{2007} & \multicolumn{2}{c|}{2009} & \multicolumn{2}{|c|}{2011} & \multicolumn{2}{c|}{2013} \\
\hline Tipo IES & Prog. & Matr. & Prog. & Matr. & Prog. & Matr. & Prog. & Matr. & Prog. & Matr. \\
\hline CRUCH & 13.5 & 28.0 & 11.3 & 23.0 & 11.2 & 21.2 & 10.2 & 15.8 & 6.9 & 13.1 \\
\hline U. Privadas & 35.7 & 36.3 & 44.6 & 42.0 & 39.0 & 35.3 & 30.8 & 32.4 & 23.6 & 29.4 \\
\hline I.P. & 44.4 & 32.4 & 36.2 & 31.1 & 41.4 & 38.6 & 51.1 & 47.5 & 61.8 & 53.2 \\
\hline C.F.T. & 6.4 & 3.3 & 8.0 & 3.9 & 8.4 & 4.9 & 8.0 & 4.3 & 7.7 & 4.3 \\
\hline Total & 171 & 15993 & 213 & 16547 & 251 & 20705 & 325 & 27466 & 377 & 31460 \\
\hline Título & & & & & & & & & & \\
\hline Prof. con Lic. & 43.9 & 60.7 & 53.0 & 64.3 & 48.2 & 54.9 & 36.9 & 46.3 & 28.9 & 41.5 \\
\hline Profesional & 39.8 & 31.8 & 29.1 & 25.7 & 29.5 & 31.2 & 34.8 & 35.6 & 38.7 & 36.9 \\
\hline Téc. Nv.Sup. & 16.4 & 7.5 & 17.8 & 10.0 & 22.3 & 13.9 & 28.3 & 18.0 & 32.4 & 21.6 \\
\hline Total & 171 & 15993 & 213 & 16547 & 251 & 20705 & 325 & 27466 & 377 & 31460 \\
\hline
\end{tabular}




\begin{tabular}{|l|c|c|c|c|c|c|c|c|c|c|}
\hline \multicolumn{10}{|l|}{ Adherencia al SUA } \\
\hline Adhiere & 35.5 & 47.5 & 24.4 & 41.6 & 27.0 & 45.8 & 30.1 & 40.0 & 27.8 & 37.5 \\
\hline No adhiere & 65.5 & 52.5 & 75.6 & 58.4 & 73.0 & 54.2 & 69.9 & 60.0 & 72.2 & 62.5 \\
\hline Total & 160 & 10288 & 196 & 10754 & 230 & 11700 & 299 & 13241 & 348 & 13380 \\
\hline Selectividad PSU \\
\hline IES Selectivas & 20.6 & 26.9 & 12.8 & 24.9 & 20.9 & 19.9 & 5.4 & 14.0 & 6.0 & 15.2 \\
\hline $\begin{array}{l}\text { IES no } \\
\text { Selectivas }\end{array}$ & 12.5 & 38.2 & 14.8 & 52.3 & 13.0 & 56.4 & 9.7 & 49.0 & 10.6 & 52.7 \\
\hline $\begin{array}{l}\text { No informa o } \\
\text { no exige }\end{array}$ & 66.9 & 34.9 & 72.4 & 22.8 & 66.1 & 23.6 & 84.9 & 37.0 & 83.3 & 32.1 \\
\hline Total & 160 & 10288 & 196 & 10754 & 230 & 11700 & 299 & 13241 & 348 & 13380 \\
\hline
\end{tabular}

Fuente: CNED - INDICES, Elaboración propia

En el área de Trabajo Social, se constata además la fuerte presencia de la formación técnica profesional tanto en IP como en CFT, situación que no es extensiva a otros ámbitos profesionales, y que incluso opera con relativa independencia del estatus de carrera universitaria que ostenta desde el 2005. En dicho año, tras un período de lucha gremial por la reivindicación del Trabajo Social, se aprobó la modificación a la Ley Orgánica Constitucional de Enseñanza conducente al restablecimiento de su exclusividad universitaria. En la práctica, se operó más bien como una pseudo-exclusividad, pues si bien, el título de Trabajador Social quedó precedido obligadamente por el grado académico de Licenciado, este marco normativo no imposibilitó la continuidad de los programas de carácter no universitario abiertos por los Institutos Profesionales con anterioridad a la promulgación de la ley. A ello se suma la proliferación de formaciones profesionales de carácter técnico con denominaciones afines, que comienzan a ser dictadas por nuevos IP y CFT. Instituciones que frente a la imposibilidad de otorgar el título profesional elaboran un conjunto de programas nominalmente diferentes al Trabajo Social, los cuales, aunque de calidad cuestionable, tienden a ser en determinados contextos laborales prácticamente equivalentes. En efecto, el título profesional con el grado de licenciado, principal certificación académica hasta el 2005, pierde progresiva vigencia hasta constituirse en la de menor participación en 2013. A costa de ellos, los programas de certificación técnica de nivel superior experimentaron un crecimiento relativo del 336\%, duplicando su participación al interior de la sub-área del Trabajo Social en su último decenio.

De igual manera, llama la atención el incremento que han tenido los programas conducentes a títulos profesionales sin licenciatura dictados por IP, los cuales duplicaron su oferta educativa en el período 2005-2013. En términos de programas, evidenciaron un crecimiento relativo del $115 \%$, y en matrícula un $68 \%$. Este incremento, dada la imposibilidad legal de que instituciones no universitarias crearan nuevos programas que otorgaran el título profesional de Trabajador Social, sería explicado por el surgimiento de un conjunto de programas de denominaciones diversas pero eventualmente homologables 
en el ámbito de desempeño laboral, en especial para la demanda educativa. En este contexto, las instituciones educativas de carácter privado han hecho gala de una exacerbada dosis de ingenio y creatividad, tanto así que no en pocos casos las denominaciones elegidas para los programas sólo operan dentro del contexto de una única institución o sede, encontrando de paso un resquicio para vulnerar el espíritu de la ley que procuraba recuperar el estatus universitario de la profesión y hacer más restrictivos los procesos formativos. En la actualidad, según datos del CNED, existirían un total de 26 denominaciones afines.

En términos generales, explorando la sub-área, los datos informan de un acelerado proceso de tecnificación de la profesión del Trabajo Social, cuyos efectos impactan decididamente en lo profesional y disciplinar. Más allá de las incuestionables implicancias en los niveles de empleabilidad y renta del ejercicio laboral, se estaría frente a un incipiente proceso de degradación disciplinar con la consiguiente pérdida de identidad respecto de lo distintivo del quehacer profesional. La atenuación de las fronteras que separan lo técnico asesor de lo profesional en el desempeño laboral, hace que el desarrollo disciplinar y el interés por fundamentar teóricamente la intervención social pierda centralidad en la labor formativa. En su defecto, dada la proliferación de denominaciones afines, el Trabajo Social se circunscribiría en el futuro cercano a una racionalidad técnica, de quehacer burocratizado y rutinario, cuyas prácticas generalistas abocadas a la acción específica contribuirían a hacer más tenues las ya difusas demarcaciones profesionales.

Por otra parte, esta heterogénea y extensa oferta educativa se implementa bajo diversas modalidades y se distribuye a través de todo el territorio nacional, en un conjunto de programas de disímiles requisitos de ingreso y cuestionables condiciones de calidad. En efecto, este crecimiento se ha hecho a costa de reducir los niveles de exigencia en la admisibilidad a los programas. Actualmente en la formación de Trabajo Social ofertada por universidades, menos de un tercio adhiere al SUA, cifra que alcanza los mínimos históricos, toda vez que en el año 2005 era de un 35,5\%, para situarse el 2013 en el 27,8\%. Así mismo, al 2013 sólo el 6\% de la oferta educativa corresponde a programas selectivos, esto es que registran al menos 500 puntos como puntaje de corte en la prueba PSU. Esta situación instala un problema de calidad al interior del sistema formativo por la vía del perfil del alumno postulante, toda vez que la evidencia informa que la rigurosidad de los procesos de admisión es un factor de preponderancia al momento de analizar los niveles de logro educativo (Wang, Coleman, \& Coley, 2003; Ávalos, 2010; Wayne, 2002; OECD, 2009; Ingersoll, 2007).

En síntesis, la oferta educativa en términos de programas y matrícula impacta decididamente sobre los niveles de stock profesionales, hetereogeniza la labor formativa con disímiles requisitos de admisión y niveles formativos, y atenúan fuertemente las ya difusas fronteras laborales, en especial, aquellos límites que otrora distinguían y separaban lo técnico de lo profesional. Problemáticas cuya resolución se constituyen hoy por hoy en un desafío histórico sin precedentes para la disciplina del Trabajo Social.

\section{CONCLUSIONES Y REFLEXIONES}

La reforma educativa de los 80', sin bien con cierto desfase temporal y heterogénea distribución espacial, refleja en cada área del conocimiento elevadas tasas de matrícula, así como un creciente número de plazas y programas formativos, impactando fuertemente en las tasas de cobertura, el stock profesional y la diversificación de la oferta educativa. 
Se trata de tendencias de un crecimiento sostenido de la oferta, que se vincula preferentemente a la formación profesional de carácter privado, y en especial en aquellas áreas del conocimiento cuya implementación de programas presenta menos restricciones. En lo que al área de las Ciencias Sociales se refiere, Psicología y Trabajo Social son los que mayor expansión registran.

La formación profesional en el área del Trabajo Social se diversifica y la oferta educativa extiende su influencia más allá del exclusivo contexto universitario, dando cuenta de un acelerado y preocupante proceso de tecnificación disciplinar.

La oferta educativa en Trabajo Social, en especial la de carácter privado, se hace más pragmática. Esto, como mecanismo de ajuste a las nuevas restricciones que la regulación del mercado educacional ha instalado a partir de la concesión de la exclusividad universitaria a la profesión. Emergen un conjunto de nuevas alternativas formativas de carácter no universitario que, aunque nominalmente distintas, en su contenido sustantivo apuntan a los mismos campos de acción en que el Trabajo Social interviene. Especial preocupación tienen aquí las denominaciones afines de la oferta de los Institutos Profesionales como forma de vulnerar el espíritu del resguardo que la ley pretendía instalar en la formación profesional del Trabajo Social.

La tecnificación disciplinar conllevaría a una formación generalista enfocada más al manejo de los procedimientos y técnicas que al dominio teórico y fundamentado de la acción. Situación que en el mediano plazo restringirá el ya menguado desarrollo disciplinar y afectará la identidad profesional, haciendo más tenues las fronteras que separan el quehacer técnico del profesional.

En lo que a la formación universitaria respecta, la proliferación de los programas de Psicología y su creciente stock de nuevos titulados ejerce presión sobre los futuros trabajadores sociales en un campo profesional compartido y de límites cada vez más difusos, acelerando un proceso de multi-competencia profesional. De esta forma, la intervención o acción social comunitaria, otrora espacio de reconocida intervención del Trabajo Social, deja de ser objeto de exclusividad identitaria profesional, e inserta problemáticas respecto de los niveles de empleabilidad, ingresos y calidad laboral.

\section{REFERENCIAS BIBLIOGRÁFICAS}

Ávalos, B. (2010). Formación inicial docente en Chile: calidad y políticas. En C. Bellei, D. Contreras, \& J. P. Valenzuela, Ecos de la revolución pingüina. Avances, debates y silencios en la reforma educacional. p. 257-284. Santiago: Pehuén Editores. Disponible en: http://www.ub.edu/obipd/ PDF\%20docs/Formaci\%C3\%B3\%20Inicial/Educaci\%C3\%B3\%20Universitaria/Documents/ Ecos\%20de\%20la\%20revolucion.pdf.

Beyer, H. (2000). Educación y desigualdad de ingresos: una nueva mirada. Estudios Públicos, (77), 97-130.

Bravo, D., \& Sanhueza, C. (2008). Is there labor market discrimination among professionals in Chile? Lawyers, doctors and businesspeople. RES Working Papers 3249. Inter - American Development Bank, Research Department.

Brunner, J. J. (2006). Diversificación y diferenciación de la educación superior en Chile en un marco internacional comparado. Documento Escuela de Gobierno, Universidad Adolfo Ibañez.

Camarena, B., \& Velarde, D. (2009). Educación superior y mercado laboral: vinculación y pertinencia social ¿Por qué? y ¿Para qué? Estudios Sociales, 105-125. 
Ingersoll, R. M. (2007). A comparative study of teacher preparation and qualifications in six nations. Philadelphia, Pa.: Graduate School of Education, University of Pennsylvania.

Iturrieta, S. (2012). Desafíos del Trabajo Social en un campo laboral con límites profesionales difusos. Katálysis, 163-172.

Meller, P. (2010). Carreras universitarias: rentabilidad, selectividad y discriminación. Santiago de Chile: uqbar.

Meller, P., \& Rappoport, D. (2003). Comparaciones internacionales de la dotación de profesionales y la posición relativa chilena. Santiago de Chile: Centro de Economía Aplicada, Universidad de Chile.

OCDE. (2009). La educación superior en Chile. Revisión de políticas nacionales de educación. Santiago de Chile: OECD y Word Bank.

Rama, C. (2009). La tendencia a la masificación de la cobertura de la educación superior en América Latina . Revista iberoamericana de educación, (50), 173-195.

Wang, A. H., Coleman, A. B., \& Coley, R. J. (2003). Preparing teachers around the world. Princeton, N. J.: Educational Testing Service.

Wayne, A. J. (2002). Teacher inequality: New evidence on disparities in teachers' academic skills. Education Policy Analysis Archives. 